\title{
7. Ausblick
}

Um die neuen Erkenntnisse über den Abbindungsmechanismus von Wassergläsern durch chemische Härtung mit Aluminium-Tetrametaphosphat zu erweitern und die Vermutungen weiter zu belegen ist es notwendig, die röntgenamorphe Binderstruktur näher zu untersuchen. Durch die fehlende strukturelle Ordnung dieser Phasen, kann mittels der Röntgendiffraktometrie keine Aussage hierzu getroffen werden. Eine Möglichkeit, den strukturellen Aufbau amorpher Systeme und Glasstrukturen zu ermitteln, stellt die Festkörper NMR-Spektroskopie dar. Da hier keine Anforderungen an geordneten Strukturen erforderlich sind, ist es möglich, die elektronische Umgebung der Kerne ${ }^{1} \mathrm{H},{ }^{27} \mathrm{Al},{ }^{29} \mathrm{Si}$ und ${ }^{31} \mathrm{P}$ durch Einpuls-Messungen zu bestimmen. Ein Abgleich der Signale aus den NMR-Spektren mit Datenbankeinträgen ist jedoch nur selten möglich, da kaum vollständige Datenbanken von anorganischen Strukturen verfügbar sind, insbesondere, wenn nur wenig über die Phasen bekannt ist. Aus den ${ }^{27} \mathrm{Al}-\mathrm{NMR}$-Untersuchungen kann auf die Koordination des Aluminiums geschlossen werden. Es ist bekannt, dass Aluminium entweder als $\mathrm{AlO}_{4}, \mathrm{AlO}_{5}$ oder $\mathrm{AlO}_{6}$ koordiniert vorkommt. Aus den ${ }^{29} \mathrm{Si}-\mathrm{Messungen}$ kann der Grad der Polykondensation der silikatischen Binderstruktur geschlossen werden und auf den Einfluss der MVZ und der Natur des Alkali-Ions die $\mathrm{Q}^{\mathrm{n}}$-Verteilung ermittelt werden. Mittels ${ }^{31} \mathrm{P}-M e s s u n g e n$ ist es möglich, Einblicke in die verschiedenen und teilweise nicht kristallinen Depolymerisationsprodukte aus der Reaktion des Wasserglases mit dem Aluminium-Tetrametaphosphat zu erhalten. Für die Bestätigung der These der mehrstufigen Depolymerisation ist es sinnvoll, die Proben nach unterschiedlichen Abbindezeiten mittels PXRD zu überprüfen. Damit ist es gegebenfalls auch möglich, eine Korrelation aus gebildeten Phasen und makroskopischen Eigenschaften, wie beispielsweise die Festigkeitssteigerung oder die Verarbeitbarkeit darzustellen. Interessant wäre zudem die Wechselwirkung mit anderen Metaphosphaten bzw. Metaphosphat-Modifikationen zu untersuchen und daraus strukturelle Wechselwirkungen aufgrund des Aufbaus des chemischen Härters zu extrahieren. Mit einer ausführlichen Untersuchung mechanischer, chemischer und thermischer Eigenschaften der verschiedenen Härtungsreaktionen ist es anschließend möglich, Aussagen über die Struktur-Eigenschafts-Korrelation zu treffen und im besten Fall Eigenschaften des Systems durch die Wahl der Edukte vorzugeben und zu steuern. 